\title{
Ações Preventivas para Úlcera por Pressão em Idosos com Declínio Funcional de Mobilidade Física no Âmbito Domiciliar*
}

\author{
Preventive Actions against Pressure Ulcers in Elderly with Functional Decline of \\ Physical Mobility at Home Environment
}

\section{Acciones Preventivas a las Úlceras por Presión en Ancianos con Declino Funcional de la Movilidad Física en el Entorno Familiar}

Josefa Danielma Lopes Ferreira', Elizabeth Souza Silva de Aguiar², Carla Lidiane Jácome de Lima',
Karen Krystine Gonçalves de Brito ${ }^{3}$, Marta Miriam Lopes Costa', Maria Júlia Guimarães Oliveira Soares

\begin{abstract}
RESUMO
As úlceras por pressão são complicações possíveis de ocorrer em pessoas em situação de fragilidade, principalmente naquelas com restrição de mobilidade e idade avançada. Este estudo teve como objetivo investigar as medidas preventivas para úlceras por pressão, utilizadas em idosos com declínio funcional de mobilidade física que vivem em domicílio. Trata-se de um estudo transversal com abordagem quantitativa, tipo inquérito domiciliar. A amostra foi composta por 25 idosos domiciliados no município de João Pessoa, na Paraíba. Foi aprovado pelo Comitê de Ética e Pesquisa do Centro de Ciências da Saúde da Universidade Federal da Paraíba, sob protocolo 124/11. Dos pesquisados, 72\% eram do sexo feminino, com faixa etária prevalente (64\%) de 80 anos ou mais; dos entrevistados, 19 (72\%) eram cuidadores informais, 6 (24\%) eram formais e apenas 1 (4\%) foi o próprio idoso. As medidas preventivas incluíram manter a pele limpa (84\%); examinar a pele diariamente (64\%); usar redistribuidores de pressão (52\%), aplicar hidratante (56\%) e usar colchão de espuma (60\%). Neste estudo, embora fossem praticadas algumas ações preventivas para úlceras por pressão, a dinâmica domiciliar encontrada apontou a necessidade de as Equipes de Saúde da Família e os serviços de atendimento domiciliares atuarem melhor quanto à prevenção de agravos, como as úlceras por pressão e suas complicações.
\end{abstract}

DESCRITORES: Estomaterapia. Enfermagem. Idoso. Úlcera por pressão. Prevenção.

\begin{abstract}
Pressure ulcers are possible complications that occur in people on fragility situations, especially those with restricted mobility and advanced age. This study aimed at investigating the preventive measures against pressure ulcers that were used in elderly people with functional decline of physical mobility who live at home. This is a cross-sectional study with a quantitative approach, of household survey type. The sample consisted of 25 elderly living in the city of João Pessoa, Paraíba state, Brazil. It was approved by the Research Ethics Committee of the Health Sciences Center from Universidade Federal do Paraíba, under protocol 124/11. A total of 72\% of the respondents was women; the most prevalent age group (64\%) was 80 years or older; 19 (72\%) of the respondents were informal caregivers, 6 (24\%) were formal and only 1 (4\%) was the elder himself. The preventive measures included keeping the skin clean (84\%), examining the skin daily (64\%), using pressure relievers (52\%), using moisturizer (56\%) and a foam mattress (60\%). In this study, although some preventive actions against pressure ulcer were taken, household dynamics pointed to the need for better performance of Family Health Teams and home care services regarding prevention of injuries, such as pressure ulcer and its complications.
\end{abstract}

DESCRIPTORS: Stomatherapy. Nursing. Elderly. Pressure ulcers. Prevention.

*Trabalho de conclusão de curso apresentado à Universidade Federal da Paraíba (UFPB) como requisito para obtenção do título de Bacharel e Licenciatura em Enfermagem, março de 2013 - João Pessoa (PB), Brasil.

'Enfermeira; Mestranda do Programa de Pós-Graduação em Enfermagem, Universidade Federal da Paraíba (UFPB) - João Pessoa (PB), Brasil. Endereço para correspondência: Rua Haroldo Monteiro Freire, 77 - José Américo de Almeida - CEP: $58073-597$ - João Pessoa (PB), Brasil -

E-mail: danielma_jp@hotmail.com

Enfermeira; Especialista em Estomaterapia; Mestre em Enfermagem pela UFPB - João Pessoa (PB), Brasil.

Enfermeira; Mestre em Enfermagem pela UFPB - João Pessoa (PB), Brasil.

4Enfermeira; Professora Doutora da Graduação e do Programa de Pós-Graduação em Enfermagem, UFPB - João Pessoa (PB), Brasil.

Artigo recebido em: 14/11/2013 - Aceito para publicação em: 09/06/2015 


\section{RESUMEN}

Las úlceras por presión son las posibles complicaciones que ocurren en personas en situaciones de fragilidad, especialmente aquellos con movilidad reducida y edad avanzada. Este estudio tuvo como objetivo investigar las medidas preventivas contra la úlcera por presión utilizadas en ancianos con declive funcional de la movilidad física, que viven en domicilios. Se trata de un estudio transversal, con abordaje cuantitativa, tipo encuesta domiciliar. La muestra se compuso por 25 ancianos que viven en la ciudad de João Pessoa, Paraíba, Brasil. El estudio fue aprobado por el Comité de Ética y Pesquisa del Centro de Ciencias de la Salud de la Universidade Federal da Paraíba, con el protocolo $124 / 11$. Los $72 \%$ de los encuestados eran mujeres, el grupo de edad más frecuente (64\%) era de 80 y más años, 19 de los encuestados (72\%) eran cuidadores informales, 6 (24\%) eran formales y sólo 1 (4\%) fue el propio anciano. Las medidas preventivas incluyeran mantener la piel limpia (84\%), examinar la piel todos los días (64\%), usar aliviadores de la presión (52\%), aplicarse la crema hidratante (56\%) y usar el colchón de espuma (60\%). En este estudio, aunque algunas acciones preventivas contra la úlcera por presión fuesen practicadas, la dinámica domiciliar encontrada señaló la necesidad de un mejor desempeño de los Equipos de Salud Familiar y los servicios de cuidado en domicilio en cuanto a la prevención de lesiones, como la úlcera por presión y suyas complicaciones.

DESCRIPTORES: Estomaterapia. Enfermería. Ancianos. Úlceras por presión. Prevención.

\section{INTRODUÇÃO}

O fato mais marcante para as sociedades atuais é o processo de envelhecimento populacional observado em todos os continentes. $\mathrm{O}$ aumento do número de idosos, tanto proporcional quanto absoluto, impõe mudanças nos modos de pensar e viver a velhice na sociedade. A longevidade alcançada pelo homem e, portanto, o aumento desta população são um tema de debates entre pesquisadores, gestores sociais e políticos de vários países do mundo ${ }^{1}$.

O Censo Populacional de 2010 do Instituto Brasileiro de Geografia e Estatística (IBGE) aponta que a sociedade brasileira caminha para o envelhecimento. Idosos com idade superior a 65 anos cresceram de 4,8\%, em 1991; 5,9\%, em 2000, para 7,4\%, em 2010. Segundo o IBGE, esse aumento é uma relação entre a ampliação da expectativa de vida e a redução abrupta da mortalidade infantil, portanto, há uma tendência a se evidenciarem mais pessoas idosas em detrimento dos mais jovens na população. Somado a esse fato, também ocorre que os brasileiros optam por constituir cada vez mais tarde menos filhos ${ }^{2}$.

O envelhecimento é um processo natural que ocorre durante o curso de vida do indivíduo e submete o corpo humano a inúmeras mudanças físicas e funcionais, com repercussões nas condições de saúde física e psicológica. Muitas dessas alterações são progressivas, como aquelas na estrutura da pele e dos músculos, as quais colocam o idoso em risco para o desenvolvimento das úlceras por pressão (UP) ${ }^{3}$.

As UP são complicações possíveis de ocorrer, principalmente, em pessoas com declínio de mobilidade física e idade avançada ${ }^{4}$. São consideradas como lesões teciduais que podem ser causadas por pressão constante, prolongada ou não, fricção, cisalhamento ou pela combinação deles. Portanto, além de a UP ocasionar dano tissular, pode provocar inúmeras dificuldades e agravar o estado clínico do paciente, especialmente naqueles com restrição na mobilização do corpo ${ }^{5}$.

Estudos nacionais relacionados à prevalência e incidência da UP apresentam índices variados quanto a sua ocorrência e/ ou presença, em função das diferentes metodologias empregadas, ao perfil dos sujeitos da amostra e aos diversificados cenários de pesquisa, tais como unidades de internação hospitalar, instituições de longa permanência para idosos e domicílios. Em um estudo realizado nas clínicas de um hospital universitário em São Paulo, identificou-se uma prevalência de 11,4\%, no primeiro momento, e 10,3\%, no segundo, o que corresponde a uma média de $10,9 \%$ de $\mathrm{UP}^{6}$. Pesquisas desenvolvidas nos domicílios de João Pessoa, na Paraîba, e Teresina, no Piauí, a partir da atenção primária com pessoas acamadas ou em cadeira de rodas, identificaram uma presença da UP de 10 e 23,52\%, respectivamente ${ }^{7}$. Ademais, as UP são consideradas problemas socioeconômico e educacional, pois o seu desenvolvimento representa um importante impacto financeiro para as instituições. Por essa razão, deve-se investir na prevenção, uma vez que os seus custos são inferiores àqueles demandados para o tratamento. O cuidado das UP ocupa o terceiro lugar em gastos em saúde, sendo ultrapassado somente por aqueles com tratamento de câncer e cirurgia cardíaca. Além disso, quando um paciente desenvolve uma UP, sua atenção demanda $50 \%$ a mais do tempo dispensado pela equipe de enfermagem, reforçando os benefícios dos cuidados preventivos tanto para o paciente e a equipe quanto pelo sistema de saúde em geral ${ }^{8}$. 
Considerando o poder incapacitante e o ônus das UP, algumas medidas de baixa tecnologia podem ser utilizadas tanto em ambientes hospitalares quanto domiciliares, tais como: promover a integridade da pele, deixando-a limpa, sem umidade e hidratada com óleos naturais ou loções hidratantes; utilizar fraldas absorventes; realizar constantemente o controle do excesso de pressão sobre as proeminências ósseas, efetivando a descompressão local e o reposicionamento com a mudança de decúbito em intervalos regulares e de forma sistemática; oferecer um adequado suporte nutricional, pois tal estado reduzido, além de prejudicar a elasticidade da pele, em longo prazo, desencadeia a anemia e reduz a oxigenação nos tecidos 9 .

Adicionalmente, é imprescindível empregar escalas de predição para o risco das UP, como as escalas de Norton, Gosnell, Waterlow e Braden, as quais são aplicadas para qualificar o diagnóstico obtido pelo raciocínio clínico, com base no de enfermagem?

De uma maneira geral, dado que compete aos serviços de saúde, por meio de suas equipes, prevenir e tratar as UP, os cuidados no processo de prevenção/tratamento/cicatrização devem ser uma preocupação constante dos profissionais e cuidadores, os quais precisam estar motivados para a busca de informações e novos recursos que permitam minimizar a morbimortalidade desencadeada por essas lesões e oferecer ao paciente a melhor assistência possível ${ }^{10}$.

Diante do exposto, partindo da continuidade de uma dissertação de Mestrado, surge a atual pesquisa, com vistas a colaborar para se repensar a assistência em saúde aos gestores e profissionais de saúde, no que concerne um planejamento adequado e uma implementação de ações para a prevenção de lesões cutâneas, tais como as UP, especialmente em pessoas idosas no âmbito domiciliar. Sendo assim, este estudo teve como objetivo investigar as medidas preventivas para UP, utilizadas em idosos com declínio funcional de mobilidade física que vivem em domicilio.

\section{MÉTODOS}

Estudo quantitativo, do tipo transversal, o qual representa a continuidade de uma investigação, mais especificamente uma dissertação de Mestrado, que foi desenvolvida na Universidade Federal da Paraíba (UFPB), sobre o risco de UP em idosos com declínio funcional de mobilidade física, residentes nos domicílios de João Pessoa, na Paraíba ${ }^{11}$. O presente estudo, portanto, refere-se à investigação das medidas preventivas para UP praticadas nesses idosos com declínio funcional de mobilidade física.
Vale destacar que tal dissertação integrou uma pesquisa maior chamada "Condições de vida e de saúde de idosos de Ribeirão Preto-SP e de João Pessoa-PB: um estudo comparado", que avaliou as condições de saúde dos idosos com idade igual ou superior a 60 anos, residentes na área urbana do município de João Pessoa, Paraíba.

$\mathrm{Na}$ busca de compreender melhor o universo amostral, haja vista que o presente estudo selecionou sua amostra partindo de outra pesquisa, a seguir, será descrito o processo desde a sua origem.

O processo amostral foi realizado por conglomerados de duplo estágio, o qual considerou a contagem da população pelo IBGE em 2007, visto que não foi possível utilizar as informações do Censo do IBGE de 2010, pois não haviam sido publicadas na ocasião do cálculo amostral. Para mensurar a amostra, foram adotados os seguintes parâmetros estatísticos: ela foi definida em 240 indivíduos, o que garantiu um erro máximo de 6,3\% com $95 \%$ de probabilidade, considerando uma população referência da cidade de João Pessoa de 61.281 idosos de 60 anos e mais $^{12}$. Para alcançar o valor de $n=240$, planejou-se o sorteio de 20 setores censitários entre os 617 existentes. Em seguida, foram visitados 12 idosos por cada um. Desses 240 sujeitos que fizeram parte do inquérito domiciliar, foram identificadas 51 pessoas com limitação física, 25 das quais apresentavam algum risco para UP, conforme a Escala de Braden, que é de risco para UP, incluindo seis parâmetros: percepção sensorial, umidade, atividade, mobilidade, nutrição, fricção e cisalhamento ${ }^{13}$. Assim, os 25 idosos citados perfizeram a amostra do presente estudo.

Os critérios de inclusão foram: pessoas com 60 anos ou mais; residentes na área urbana de João Pessoa, Paraíba; que apresentavam algum nível de risco para UP segundo a Escala de Braden; escore $\leq 18$ - índices iguais ou menores que 18 classificam as pessoas em níveis de risco para UP. Em suma, o não atendimento a um desses critérios implicou na não inclusão de idosos na amostra.

Durante abril e junho de 2011, transcorreu a coleta inicial dos dados, a qual identificou os idosos com declínio funcional de mobilidade física com algum risco para UP segundo a Escala de Braden aplicada; em seguida, entre outubro de 2011 a janeiro de 2012, as pesquisadoras retornaram aos domicílios para pesquisar as medidas preventivas para UP praticadas com os idosos estudados. Para tanto, utilizou-se a técnica de entrevista com o idoso e/ou cuidador, além de observação não participativa. Para essa etapa, utilizou-se um instrumento elaborado pelas pesquisadoras, que passou por pré-teste e abordou aspectos sociodemográficos e cuidados para a prevenção de UP. 
Os dados foram duplamente digitados e validados em uma planilha do programa Microsoft Excel, e os cálculos estatísticos foram realizados no software SPSS (Statistical Package for the Social Sciences), versão 20.0.

A pesquisa foi submetida à apreciação do Comitê de Ética e Pesquisa do Centro de Ciências da Saúde da UFPB, sob protocolo 124/11.

\section{RESULTADOS}

Dentre os 25 idosos pesquisados, a maioria dos investigados, 18 (72\%), era do sexo feminino; quanto à idade, 16 (64\%) estavam na faixa etária de 80 anos ou mais; seis deles (24\%) tinham de 70 a 79 anos, e apenas três (12\%) estavam com 60 a 69 anos. A cor da pele predominante foi a branca, com um total de 15 (64\%). Verificou-se que todos tinham cuidador domiciliar, sendo 19 (72\%) deles informais, como familiares, e seis (24\%) formais. A presença de tal profissional deve-se ao perfil desses idosos, visto que são pessoas com declínio de mobilidade física e a maioria dependente de cuidados, portanto se tratava de pessoas com dificuldades para deambular, usavam cadeiras de rodas ou ainda estavam totalmente acamadas.

Quanto às condições de saúde identificadas, merecem destaque: a doença neurológica (especificamente na maioria dos idosos com 80 anos ou mais), o acidente vascular encefálico, a incontinência urinária e/ou fecal, as osteoartrites, a hipertensão arterial sistêmica e a visão prejudicada.

Concernente às medidas preventivas implementadas no domicílio para evitar ou retardar o desenvolvimento de UP, as mais frequentes foram: manter a pele limpa (84\%), examinar a pele diariamente (64\%) e usar aliviadores de pressão (52\%), conforme a descrição na Tabela $1^{14}$.

Os idosos acamados e em uso das cadeiras de rodas, ou aqueles incapazes de se posicionarem, são mais propensos para a formação da UP, portanto devem receber atenção específica e sistemática a fim de evitar fatores adicionais de riscos que resultem em lesões nos tecidos. Logo, no presente estudo, 21 (84\%) não realizavam a movimentação dos membros inferiores (MMII), 21 (84\%) não efetivavam a descompressão local, 20 (80\%) não faziam o reposicionamento da pessoa sentada ou em uso cadeiras de rodas.

Com relação aos produtos utilizados após a higienização da pele, os mais citados foram as loções hidratantes, utilizadas por 14 (56\%), como mostra a Tabela 2.

Também foi possível investigar as superfícies de suporte utilizadas pelos idosos pesquisados, conforme apresentado na Tabela 3 , em que se destaca o uso do colchão de espuma por 15 participantes (60\%).

Tabela 1. Medidas preventivas utilizadas para úlcera por pressão nos idosos que vivem em domicílios (n-=25). João Pessoa, Paraíba, 2011/2012.

\begin{tabular}{|c|c|c|c|c|}
\hline \multirow{2}{*}{ Variável } & \multicolumn{2}{|c|}{ Sim } & \multicolumn{2}{|c|}{ Não } \\
\hline & $\mathrm{n}(\%)$ & IC95\% & $\mathrm{n}(\%)$ & IC95\% \\
\hline Massagem de conforto** & $1(4)$ & $0-12$ & $24(96)$ & $88-100$ \\
\hline Elevação da cabeceira a $30^{\circ}$ & $3(12)$ & $0-28$ & $22(88)$ & $72-100$ \\
\hline Lençóis sem rugas, pregas ou sujidade & $8(32)$ & $16-48$ & $17(68)$ & $52-84$ \\
\hline $\begin{array}{l}\text { Uso de lençol móvel ou forro da cama para } \\
\text { movimentar o idoso acamado }\end{array}$ & $1(4)$ & $0-12$ & $24(96)$ & $88-100$ \\
\hline Alimentação equilibrada & $5(20)$ & $4-36$ & $20(80)$ & $64-96$ \\
\hline Pele limpa & $21(84)$ & $68-96$ & $4(16)$ & $4-32$ \\
\hline Uso dos redistribuidores de pressão & $13(52)$ & $32-72$ & $12(48)$ & $28-68$ \\
\hline Exame da pele diariamente & $16(64)$ & $44-84$ & $9(36)$ & $16-56$ \\
\hline Elevação dos membros inferiores & $4(16)$ & $4-32$ & $21(84)$ & $68-96$ \\
\hline Levantar para descompressão local & $4(16)$ & $4-28$ & $21(84)$ & $72-96$ \\
\hline Mudança de decúbito & $1(4)$ & $0-12$ & $24(96)$ & $88-100$ \\
\hline $\begin{array}{l}\text { Reposicionamento da pessoa sentada ou em cadeiras } \\
\text { de rodas }\end{array}$ & $5(20)$ & $4-40$ & $20(80)$ & $60-96$ \\
\hline
\end{tabular}

IC: intervalo de confiança; *Embora essa ação esteja apresentada na tabela, destacamos que ela é contraindicada sobre as proeminências ósseas e/ou áreas hiperemiadas, na presença de inflamação aguda e onde existe a possibilidade de vasos sanguíneos danificados ou pele frágil ${ }^{14}$. 
Tabela 2. Uso de soluções para manter a integridade da pele em idosos que vivem em domicílios (n=25). João Pessoa, Paraíba, 2011/2012.

\begin{tabular}{|c|c|c|c|c|}
\hline \multirow{2}{*}{ Variável } & \multicolumn{2}{|c|}{ Sim } & \multicolumn{2}{|c|}{ Não } \\
\hline & n (\%) & IC95\% & $\mathrm{n}(\%)$ & IC95\% \\
\hline Óleos vegetais & $6(24)$ & $8-40$ & $19(76)$ & $60-92$ \\
\hline $\begin{array}{l}\text { Loções } \\
\text { hidratantes }\end{array}$ & $14(56)$ & $36-76$ & $11(44)$ & $24-64$ \\
\hline Outros* & $7(28)$ & $12-48$ & $18(72)$ & $52-88$ \\
\hline
\end{tabular}

*Pomadas, soluções caseiras, pasta d'água; IC: intervalo de confiança.

Tabela 3. Superfícies de suporte utilizadas pelos idosos. João Pessoa, Paraíba, 2011/2012.

\begin{tabular}{|c|c|c|c|c|}
\hline \multirow{2}{*}{ Variável } & \multicolumn{2}{|c|}{ Sim } & \multicolumn{2}{|c|}{ Não } \\
\hline & n (\%) & IC95\% & n (\%) & IC95\% \\
\hline & $15(60)$ & $40-80$ & $10(40)$ & $20-60$ \\
\hline Colchão de ar* & $2(8)$ & $0-20$ & $23(92)$ & $80-100$ \\
\hline $\begin{array}{l}\text { Colchão de } \\
\text { água }\end{array}$ & $1(4)$ & $0-12$ & $24(96)$ & $88-100$ \\
\hline $\begin{array}{l}\text { Colchão de } \\
\text { caixa de ovo }\end{array}$ & $6(24)$ & $8-40$ & $19(76)$ & 60-92 \\
\hline Rede ${ }^{\star \star}$ & $1(4)$ & $0-12$ & $24(96)$ & $88-100$ \\
\hline
\end{tabular}

*A superfície citada refere-se ao colchão de ar estático; **Culturalmente usadas por sertanejos no Nordeste; IC: intervalo de confiança.

\section{DISCUSSÃO}

Estudos nacionais apontam o sexo feminino como o grupo de maior sobrevida em detrimento do masculino, em virtude do autocuidado, especialmente com a saúde, praticado por elas ${ }^{15,16}$. Os homens não têm a cultura da prevenção, uma vez que não procuram os serviços de saúde com a mesma frequência que as mulheres, pois é um costume masculino não demonstrar a necessidade de cuidados, já que, de forma consciente ou inconsciente, essa é uma característica fraca, e como tal, não combina com a concepção masculina de homem forte e viril ${ }^{17}$.

Quanto à idade, verificaram-se diferenças percentuais nas faixas etárias, com o maior risco para UP entre os idosos octogenários ou com mais de 80 anos. Pesquisas referem que existe um relacionamento linear entre o aumento da idade dos pacientes e da ocorrência de UP, pois, além da própria fisiologia do envelhecimento, a prevalência das condições crônicas que ocorrem nessa época da vida leva à diminuição da capacidade funcional nos idosos, e essas situações somadas contribuem para elevar o risco de UP ${ }^{15,16}$.

Cuidar de um idoso no domicílio é uma tarefa árdua. $\mathrm{Na}$ maioria das vezes, é um papel desempenhado por familiares, especialmente do gênero feminino, como esposa, filha, neta ou irmã. Em um estudo realizado em Pelotas, no estado do Rio Grande do Sul, os familiares eram responsáveis pelo cuidado domiciliar com os idosos, desempenhado, principalmente, pelo esposo/a. Estudos mostram que é crescente a sua necessidade, sendo que, entre os cuidadores, prevalecem os familiares (cônjuge, filhos, irmãos etc.), também chamados de informais, em detrimento dos cuidadores formais ${ }^{18}$.

Vale destacar que cuidadores são aqueles que prestam cuidados ao idoso dependente e podem ou não ter vínculo familiar. Existem dois tipos: o cuidador formal e o informal. O primeiro é um profissional preparado em uma instituição de ensino para prestar cuidados no domicílio, segundo as necessidades do idoso. Por outro lado, o informal advém da dependência e necessidade de cuidados de um familiar, amigo, vizinho ou outros grupos de pessoas, o qual não é economicamente remunerado pela atenção desenvolvida ${ }^{19}$.

Neste estudo, também foram encontrados problemas de saúde, como déficits cognitivos determinados pela senilidade e pelas doenças de base, além de distúrbios cardiovasculares, neurológicos e degenerativos que trazem diversas consequências, desde a inabilidade de autocuidado até perdas sensitivas e de mobilidade física, as quais, juntas, predispõem a pessoa ao risco de UP, assim como o surgimento de complicações, uma vez que a lesão representa uma porta de entrada para infecções ${ }^{11}$. As medidas preventivas têm custos, porém os gastos com a hospitalização ou o prolongamento do tempo de tratamento das lesões são superiores. Várias medidas preventivas podem ser adotadas para evitar o desenvolvimento da UP, bem como o emprego dos instrumentos de avaliação e predição de risco, tais como as várias escalas validadas, a exemplo a Escala de Braden, utilizada no presente traba1 ho ${ }^{9}$. Partindo desse princípio, existem medidas preventivas que podem ser realizadas no domicílio do idoso em risco de UP, algumas apresentadas na Tabela 1 , as quais foram investigadas e serão discutidas adiante.

Dentre as ações de prevenção encontradas, houve maior prevalência nas medidas relacionadas à manutenção de higiene cutânea (84\%) e ao exame físico cutâneo (64\%). A pele seca pode ser um sinal de desidratação, que se caracteriza pela diminuição de água, perda de eletrólitos totais do organismo, pele com elasticidade diminuída e pouca tolerância ao calor, à fricção e à pressão, tornando-a suscetível à 
ruptura. Assim, examinar a pele e manter a sua higienização adequada são medidas simples que podem diminuir o risco de UP. Hidratar a pele seja com produtos utilizados após o banho ou a troca de fraldas ou o simples ato de ingerir água também ajudam ${ }^{18}$. Um estudo com abordagem de caráter qualitativo, realizado em uma Unidade de Terapia Intensiva (UTI) adulto de um hospital público do município de Chapecó, em Santa Catarina, mostra que o banho é o melhor momento para examinar a pele e avaliar a eficácia de outras medidas preventivas 9 .

No que se refere à elevação da cabeceira a $30^{\circ}$, a manutenção dos lençóis sem rugas, pregas ou sujidade e ao uso de lençol móvel ou forro da cama para movimentar o idoso acamado, poucos, respectivamente 12,32 e $4 \%$, empregavam essas medidas no cotidiano. Outros estudos indicaram que atos importantes, como o uso do lençol móvel, a elevação da cabeceira a $30^{\circ}$ e a manutenção dos lençóis limpos, sem pregas ou rugas, também foram pouco utilizados pelos idosos pesquisados ${ }^{16}$. Não se deve elevar o leito da cabeceira da cama acima dos $30^{\circ}$ por dificultar os retornos dos fluxos sanguíneos e linfáticos e favorecer a fricção e/ou o cisalhamento da pele ${ }^{14}$.

Quanto à mudança de decúbito, à descompressão local, ao reposicionamento de pessoa sentada ou em cadeiras de rodas e à elevação dos MMII, também foram ações poucos efetivadas pelos pesquisados. Estudos apresentam que a mudança de decúbito é uma conduta importante para a prevenção de UP, que deve ser realizada a cada duas horas em indivíduos acamados. Essas são importantes para diminuir-se o risco. Já para os que permanecem sentados por períodos longos, o reposicionamento deve ser realizado a cada hora e com a proteção de almofadas de gel ou ar, porém dispositivos do tipo roda d'água não devem ser empregados, pois as mudanças de posição frequentes e o uso de almofadas reduzem a pressão em proeminências ósseas ${ }^{20,21}$.

O reposicionamento de pessoa sentada ou em cadeiras de rodas, por sua vez, alterna ou alivia a pressão sobre áreas suscetíveis e diminui o risco do desenvolvimento de UP. Travesseiros e coxins são materiais facilmente disponíveis e que podem ser utilizados para auxiliar a redistribuição da pressão, por exemplo, na elevação dos MMII. Quando utilizados de forma apropriada, podem expandir a superfície que suporta o peso. Geralmente, a pele de pacientes com risco para UP rompe-se facilmente durante o reposicionamento, portanto, deve-se tomar cuidado com a fricção durante este procedimento ${ }^{14}$.
A alimentação estava pouco equilibrada nos idosos investigados, apenas $20 \%$ tinham essa preocupação. Sabe-se que a análise do índice de massa corporal (IMC) acima e abaixo da média representava risco para o desenvolvimento de UP. Em pacientes emagrecidos, em que a quantidade de gordura é mínima nas proeminências ósseas, é mais propensa a maior pressão sobre essas áreas. Porém, se o paciente é obeso, também se mantém um risco para o desenvolvimento da úlcera em função da restrição de mobilidade física ${ }^{16}$. Acrescente-se, ainda, a importância de proporcionar suporte nutricional adequado, pois o estado nutricional reduzido, além de prejudicar a elasticidade da pele em longo prazo, leva à anemia e diminuição de oxigênio nas células 9 .

No que diz respeito ao uso de soluções para manter a integridade da pele dos idosos (Tabela 2), as loções hidratantes foram as mais utilizadas (56\%). Os hidratantes devem ser aplicados suavemente, e a pele deve ser limpa frequentemente com água morna e um produto de limpeza neutro, sem sabonete, pois este provoca ressecamento da pele. Essas intervenções, como o ato de aplicar hidratante e examinar a pele durante o banho ou a troca de fralda, por exemplo, refletem o processo de estar atento e identificar precocemente fatores de risco para UP'. Durante a hidratação da pele, não devem ser massageadas áreas de proeminências ósseas ou hiperemiadas. A aplicação de hidratante deve ser realizada com movimentos suaves e circulares ${ }^{14}$.

Medidas como controle do excesso de pressão sobre as proeminências ósseas podem ser empregadas, protegendo-as com travesseiros e almofadas de espuma ou colchões de espuma, ar estático e dinâmico, gel e água, pois redistribuem o peso e reduzem a pressão. São também chamados de aliviadores de pressão, apresentados na Tabela 3. Neste estudo, o colchão de espuma foi o mais utilizado pelos idosos (60\%), sendo que, muitas vezes, estava associado ao uso do colchão de ar ou caixa de ovo. Superfícies de apoio específicas, como colchões, redistribuem a pressão que o corpo do paciente exerce sobre a pele e os tecidos subcutâneos. Se a mobilidade do paciente está comprometida e a pressão nessa interface não é redistribuída, esta poderá prejudicar a circulação, levando ao surgimento da úlcera ${ }^{14}$.

Esses resultados evidenciam as características do paciente e do processo de cuidar; entretanto, aspectos referentes à estrutura familiar e àquela oferecida pelos serviços para a assistência domiciliária precisam ser investigados mais atentamente, de forma a se compreender mais o problema. 


\section{CONCLUSÃO}

Nesta pesquisa, foram identificadas as seguintes ações prioritárias de medidas preventivas para prevenir as UP: manter a pele limpa, examiná-la e hidratá-la diariamente e utilizar redistribuidores de pressão. Ressalta-se que, dentre as diversas ações para prevenção de UP, houve predominância de três ações. Portanto, conclui-se que a maioria dos pesquisados não utilizava as medidas preventivas de forma eficaz, muito embora, neste estudo, $100 \%$ deles demonstrassem risco para desenvolver a lesão. Assim, infere-se que os cuidadores não tinham o preparo adequado para a realização dessas medidas, seja por falta de conhecimentos ou condições financeiras precárias.

Esse apontamento remete que, na dinâmica domiciliar, as Equipes de Estratégias de Saúde da Família e os serviços de atendimento domiciliares devem melhorar a sua atuação quanto à prevenção de agravos, como as UP e as suas complicações. Como é crescente a população de pessoas idosas que necessitam de cuidados especiais, é imprescindível que os profissionais de saúde, especialmente os enfermeiros, atuem na capacitação dos cuidadores, para evitar outros agravos à saúde dos idosos e conferir qualidade de vida melhor para eles.

\section{REFERÊNCIAS}

1. Brasil. Ministério da Saúde. Secretaria de Atenção à Saúde. Departamento de Ações Programáticas e Estratégicas. Atenção à saúde da pessoa idosa e envelhecimento. Brasília; 2010. 44p.

2. Brasil. Instituto Brasileiro de Geografia e Estatística. Sinopse do Censo Demográfico 2010. São Paulo: IBGE; 2011. [citado 21 jul. 2014]. Disponível em: http://www.ibge.gov.br/home/ estatistica/populacao/censo2010/tabelas_pdf/Paraiba.pdf

3. Lima AP, Delgado El. A melhor idade do Brasil: aspectos biopsicossociais decorrentes do processo de envelhecimento. Rev Ulbra Movimento. 2010;1(2):76-91.

4. Freitas MC, Medeiros AB, Guedes MV, Almeida PC, Galiza FT, Nogueira JM. Úlcera por pressão em idosos institucionalizados: análise da prevalência e fatores de risco. Rev Gaúcha Enfermagem. 2011;32(1):143-50.

5. European Pressure Ulcer Advisory Panel (EPUAP), National Pressure Ulcer Advisory Panel (NPUAP). New 2014 Prevention and Treatment of Pressure Ulcers: Clinical Practical Guideline. Europa; EUA; 2009. [citado 20 jul. 2014]. Disponível em: http://www.npuap.org/wp-content/ uploads/2012/02/Final_Quick_Prevention_for_web_2010.pdf

6. da Silva Cardoso JR, Blanes L, Augusto Calil J, Ferreira Chacon JM, Masako Ferreira L. Prevalence of pressure ulcers in a Brazilian Hospital: results of a cross-sectional study. Ostomy Wound Manage. 2010;56(10):52-7.

7. Ferreira JD, Aguiar ES, Soares MJ, Silva MA, Oliveira SH. Risco e prevalência de úlcera por pressão em usuários da rede básica de saúde. Rev Enferm UFPE Online. 2012;6(9):2045-51.

8. Serpa LF, Santos VL, Campanili TC, Queiroz M. Validade preditiva da Escala de Braden para o risco de desenvolvimento de úlcera por pressão em pacientes críticos. Rev Latino-Am Enfermagem. 2011;19(1):1-8.

9. Lise F, Silva LC. Prevenção de úlcera por pressão: instrumentalizando a enfermagem e orientando o familiar cuidador. Acta Sci Health Sci. 2007;29(2):85-9.

10. Mattia AL, Rocha AM, Barbosa MH, Guimarães MA, Borgato MO, Silva SR, Freitas Filho JP. Úlcera por pressão em UTI: fatores de risco e medidas de prevenção. Saúde Col. 2010;7(46):296-9

11. Aguiar ES, Soares MJ, Caliri MH, Costa MM, Oliveira SH. Avaliação da capacidade funcional de idosos associada ao risco de úlcera por pressão. Acta Paul Enferm. 2012;25(Número Especial 1):94-100.

12. Brasil. Instituto Brasileiro de Geografia e Estatística. Censo Demográfico 2007. São Paulo: IBGE; 2007. [citado 21 jul. 2014. Disponível em: http://www.ibge.gov.br/home/ estatistica/populacao/contagem2007/

13. Braden B, Bergstrom N. A conceptual schema for the study of the etiology of pressure sores. Rehab Nurs. 1987;12(1):8-12.

14. Brasil. Ministério da Saúde. Anvisa. Fiocruz. Protocolo para prevenção de úlcera por pressão. Brasília; 2013. [citado 30 jul. 2014]. Disponível em: http://www.hospitalsantalucinda. com.br/downloads/prot_prevencao_ulcera_por_pressao.pdf

15. Chayamiti EM, Caliri MH. Úlcera por pressão em pacientes sob assistência domiciliária. Acta Paul Enferm. 2010;23(1):29-34.

16. Fernandes MG, Costa KN, Santos SR, Pereira MA, Oliveira DS, Brito SS. Risco para úlcera por pressão em idosos hospitalizados: aplicação da Escala de Waterlow. Rev Enferm UERJ. 2012;20(1):56-60.

17. Fernandes LA. O enfermeiro atuando na prevenção das úlceras de pressão. Rev Eletrônica Cuatrimestral Enferm. 2009 [citado 10 jul. 2014];13. Disponível em: http//:www.um.es/eglobal/

18. Duca GF, Thumé E, Hallal PC. Prevalência e fatores associados ao cuidado domiciliar a idosos. Rev Saúde Pública. 2011;45(1):113-20.

19. Cruz DC, Loureiro HA, Silva MA, Fernandes MM. As vivências do cuidador informal do idoso dependente. Rev Enferm Referência. 2010;3(2):127-36.

20. Goes KS, Mabda M, Almeida DT. Úlcera por pressão: um desafio na prática profissional do enfermeiro de Unidade de Terapia Intensiva. Rev Estima. 2007;5(3):29-35.

21. Coêlho AD, Lopes MV, Melo RP, Castro ME. O idoso e a úlcera por pressão em serviço de atendimento domiciliar. Rev Rene. 2012;13(3):639-49. 